\title{
Hábitos alimentares de serpentes em Espigão do Oeste, Rondônia, Brasil
}

\author{
Paulo Sérgio Bernarde ${ }^{1,3}$ \& Augusto Shinya Abe ${ }^{2}$ \\ ${ }^{1}$ Laboratório de Herpetologia, Centro Multidisciplinar, Campus Floresta, Universidade Federal do Acre - UFAC, \\ CEP 69980-000 Cruzeiro do Sul, AC, Brasil \\ ${ }^{2}$ Departamento de Zoologia, Instituto de Biociências, Universidade Estadual Paulista - UNIP, \\ CP 199, CEP 13506-900 Rio Claro, SP, Brasil. \\ ${ }^{3}$ Autor para correspondência: Paulo Sérgio Bernarde, email: snakebernarde@hotmail.com
}

BERNARDE, P.S. \& ABE, A.S. Food habits of snakes from Espigão do Oeste, Rondônia, Brazil. Biota Neotrop. 10(1): http://www.biotaneotropica.org.br/v10n1/en/abstract?article+bn02510012010.

\begin{abstract}
The present study reports on food habits of snakes in Espigão do Oeste, Rondônia State (Southwestern Amazonia), Brazil. Snakes were recorded by time constrained search, pitfall traps with drift fences, captures by local inhabitants, and opportunistic sightings. The snakes collected had their stomachs and intestinal contents examined. Information on food habits was obtained for 89 specimens belonging to 31 species. The majority of the 114 items found (stomach contents and field observations) were adult frogs (38\%), followed by mammals (16\%), lizards (15\%), mollusks (13\%), birds (5\%), and snakes (5\%). The majority of snake species from Espigão do Oeste feed on lizards, as in Central Amazonia (Manaus). A smaller proportion of snakes in the Amazonia feed upon frogs in relation to the Pantanal, South and Southeastern Brazil. These differences may be due to greater proportion of Xenodontinae in extra-Amazonian communities, as many snakes in that clade prey upon frogs. Most of the frogs and lizards species recorded in stomach contents snakes are terrestrial, which should be associated with most species of analysed snakes (52\%) foraging on the ground.
\end{abstract}

Keywords: Reptilia, Squamata, prey, diet, Amazonia.

BERNARDE, P.S. \& ABE, A.S. Hábitos alimentares de serpentes em Espigão do Oeste, Rondônia, Brasil. Biota Neotrop. 10(1): http://www.biotaneotropica.org.br/v10n1/en/abstract?article+bn02510012010.

Resumo: O presente estudo teve como objetivo estudar os hábitos alimentares das serpentes em Espigão do Oeste, Rondônia (Sudoeste da Amazônia), Brasil. As serpentes foram registradas através de procura limitada por tempo, armadilhas de interceptação e queda, coleta por terceiros e encontros ocasionais. As serpentes coletadas tiveram o conteúdo estomacal e intestinal examinados. Em 89 espécimes pertencentes a 31 espécies de serpentes foram registradas informações sobre alimentação. Dos 114 itens registrados (conteúdos estomacais e observações na natureza), a maioria foi de anuros (38\%), seguidos de mamíferos (16\%), lagartos (15\%), moluscos (13\%), aves $(5 \%)$ e serpentes (5\%). A maioria das espécies de serpentes de Espigão do Oeste preda lagartos, assim como na Amazônia Central (Manaus). Uma menor proporção de serpentes na Amazônia utiliza anuros em relação às regiões do Pantanal, sul e sudeste do Brasil. Essas diferenças podem ser devidas à maior proporção de Xenodontinae nas comunidades extra-amazônicas, uma vez que muitas das serpentes desse clado predam anuros. A maioria dos anfíbios anuros e lagartos encontrados nos conteúdos estomacais das serpentes apresentam hábitos terrícolas, o que deve estar associado com a maioria das espécies de serpentes (52\%) forragearem sobre o chão.

Palavras-chave: Reptilia, Squamata, presas, dieta, Amazônia. 


\section{Introdução}

Diferentemente de anuros e lagartos, em que a maioria das espécies alimenta-se de artrópodos, as serpentes apresentam dieta muito diversificada (Greene 1997) sendo a dieta um dos principais eixos no nicho deste grupo (Toft 1985). Serpentes são animais carnívoros, que se alimentam de uma ampla variedade de presas, como minhocas, moluscos, onicóforos, aranhas, quilópodos, insetos, crustáceos, peixes, gimnofionos, anuros, lagartos, outras serpentes, tartarugas, crocodilianos, pássaros, ovos, roedores, marsupiais, morcegos (Greene 1997, Martins \& Oliveira 1998). Apesar da importância do conhecimento da dieta das serpentes para que sejam estabelecidas guildas nas análises ecológicas das comunidades (e.g. Vitt 1983, Cadle \& Greene 1993, Martins \& Oliveira 1998, Bernarde \& Abe 2006), informações sobre as espécies na região Neotropical ainda são incipientes (Zanella \& Cechin 2009). Informações específicas sobre os tipos de presas consumidas por cada espécie são necessárias para que se possa comparar a dieta das serpentes pertencentes a uma mesma guilda (e.g. Vitt 1983, Zanella \& Cechin 2009), assim como informações sobre o tipo de substrato utilizado e período de atividade de forrageio (e.g. Oliveira \& Martins 2001, Martins et al. 2002, França et al. 2008).

São conhecidas mais de 149 espécies de serpentes para a Amazônia brasileira (Ávila-Pires et al. 2007) e faltam informações sobre os hábitos alimentares para grande parte das espécies. Uma das espécies que tem a dieta mais conhecida é o viperídeo Bothrops atrox (Martins \& Gordo 1993, Egler et al. 1996, Martins \& Oliveira 1998, Oliveira \& Martins 2001, Macedo-Bernarde \& Bernarde 2005, Nascimento et al. 2008), provavelmente por ser uma das serpentes mais abundantes (Oliveira \& Martins 2001). Muito do que existe sobre o conhecimento da dieta de serpentes da Amazônia refere-se a espécies nas regiões leste (Cunha \& Nascimento 1993) e central (Martins \& Oliveira 1998).

Em Rondônia, informações sumarizadas sobre a dieta de serpentes constam de estudo sobre comunidade realizado em Espigão do Oeste por Bernarde \& Abe (2006). Informações adicionais constam de notas curtas (Macedo-Bernarde \& Bernarde 2005, Macedo-Bernarde 2006, Bernarde et al. 2008, Nascimento et al. 2008) ou registros anedóticos (Vanzolini 1986, Turci \& Bernarde 2008). O presente estudo tem como objetivo apresentar informações mais detalhadas sobre os hábitos alimentares de serpentes em Espigão do Oeste, Rondônia (Sudoeste da Amazônia), Brasil.

\section{Material e Métodos}

A área de estudo compreende o Município de Espigão do Oeste (11 $30^{\circ} \mathrm{S}$ e $60^{\circ} 40^{\prime} \mathrm{O}$ ) localizado no Sudoeste da Amazônia, estado de Rondônia, Brasil (Figura 1). A altitude da região varia em torno de $280 \mathrm{~m}$. A região apresenta média pluviométrica anual de $2300 \mathrm{~mm}$, com um período de seca entre abril e setembro (dados fornecidos pela Prefeitura Municipal). A temperatura média anual é de $26{ }^{\circ} \mathrm{C}$. A vegetação enquadra-se dentro do Domínio Equatorial Amazônico -“Amazônia" (Ab'Saber 1977), sendo representada pela Floresta Ombrófila Aberta Submontana. O município teve início de sua colonização na Década de 70, com desmatamento principalmente para atividade de pecuária (Oliveira 2002). As áreas de pastagem são caracterizadas por gramíneas artificiais (Brachiaria brizantha, Panicum maximum), com poucos arbustos e árvores.

As informações sobre os hábitos alimentares (conteúdos estomacais e observações na natureza) sobre as serpentes foram obtidas durante estudo mais amplo sobre a comunidade local (ver Bernarde \& Abe 2006), entre fevereiro de 2001 e dezembro de 2002, e em seis visitas anteriores (julho de 1994, janeiro e julho de 2005, janeiro de 1996, janeiro de 1997 e janeiro de 1998). Utilizou-se os métodos de

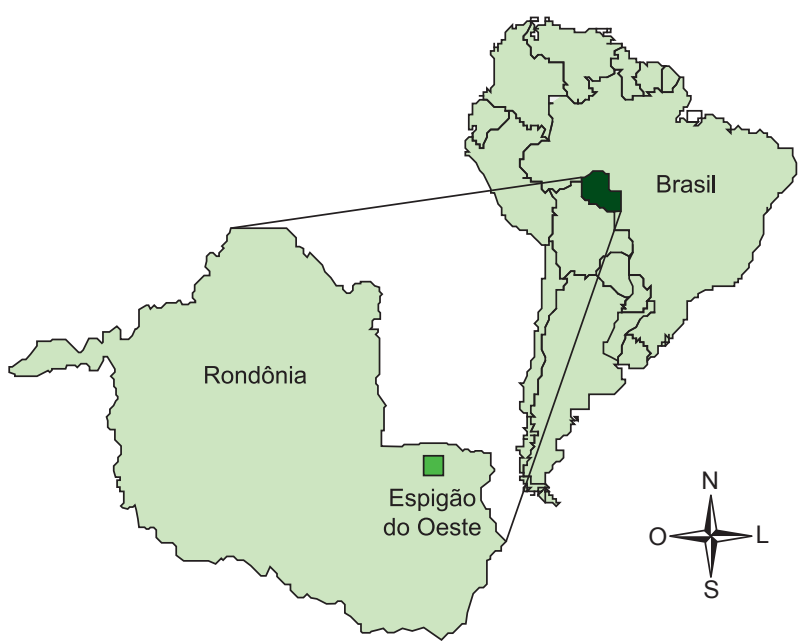

Figura 1. Mapa da América do Sul mostrando a localização do Município de Espigão do Oeste no estado de Rondônia, Brasil.

Figure 1. Map of South America showing the localization of Municipality of Espigão do Oeste in Rondônia State, Brazil.

procura limitada por tempo, armadilhas de interceptação e queda, coleta por terceiros e encontros ocasionais em ambientes de floresta e de pastagem. A procura regular de serpentes e as capturas em armadilhas de interceptação e queda foram realizadas na Fazenda Jaburi $\left(11^{\circ} 35^{\prime}-11^{\circ} 38^{\prime} \mathrm{S}\right.$ e $\left.60^{\circ} 41^{\prime}-60^{\circ} 45^{\prime} \mathrm{O}\right)$ durante os meses de abril de 2001 a março de 2002. Esta propriedade rural situa-se no Km 32 da Rodovia do Calcário, compreendendo uma área de 4000 ha, dos quais $50 \%$ correspondem à reserva legal.

Os espécimes coletados durante este estudo foram depositados nas seguintes coleções herpetológicas: Instituto Butantan (IB), São Paulo, SP; Museu Paraense Emílio Goeldi (MPEG), Belém, PA; Instituto Nacional de Pesquisa da Amazônia (INPA), Manaus, AM; Universidade Estadual de Londrina (MZUEL), Londrina, PR; Museu de História Natural Capão da Imbuia (MHNCI), Curitiba, PR.

De 462 espécimes de serpentes pertencentes a 56 espécies registradas em Espigão do Oeste por Bernarde \& Abe (2006), 282 tiveram o conteúdo estomacal e intestinal examinados. Os itens alimentares encontrados foram identificados até os seus grandes níveis taxonômicos (moluscos, roedores e pássaros) ou até o nível de gênero ou espécie (anuros, lagartos e serpentes) utilizando-se chaves taxonômicas e guias de identificação (ver relação em Bernarde \& Abe 2006, Bernarde 2007, Macedo et al. 2008). Sempre que a presa foi proveniente de uma serpente capturada em armadilha de interceptação e queda, isto foi mencionado, dada a possibilidade da serpente ter ingerido alguma presa que também tenha caído na armadilha (Cechin \& Martins 2000) e que não faça parte de sua dieta usual. Abreviaturas utilizadas são CRC (comprimento rostro-cloacal) e CC (comprimento da cauda).

\section{Resultados}

Dentre os 282 espécimes de serpentes que tiveram seus conteúdos estomacais analisados, 82 (29\%), pertencentes a 30 espécies distintas, apresentaram presas. Sete registros de serpentes predando ou perseguindo animais ou regurgitando presas também forneceram informações sobre alimentação, totalizando 114 itens registrados para 89 espécimes pertencentes a 31 espécies de serpentes. Dos 114 itens registrados, a maioria foi de anuros $(38 \%)$, seguido de mamíferos (16\%), lagartos (15\%), moluscos $(13 \%)$, aves $(5 \%)$ e serpentes $(5 \%)$ (Tabela 1). 
Tabela 1. Registros sobre hábitos alimentares encontrados em serpentes de Espigão do Oeste, Rondônia. N = quantidade de serpentes com o respectivo conteúdo estomacal ou observação.

Table 1. Food habits records found in snakes from Espigão do Oeste, Rondônia. $\mathrm{N}$ = quantity of snakes with the respective stomacal content or observation.

\begin{tabular}{|c|c|c|}
\hline Família/ Espécie & $\mathbf{N}$ & Conteúdo estomacal/ Observação \\
\hline \multicolumn{3}{|l|}{ BOIDAE } \\
\hline \multirow{4}{*}{ Boa constrictor Linnaeus, 1758} & 1 & Ameiva ameiva (Sauria Teiidae) \\
\hline & 2 & Fragmentos de roedor \\
\hline & 1 & Volatinia jacarina (Aves Passeriformes) + roedor \\
\hline & 1 & Fragmentos de roedor e de ave \\
\hline \multirow[t]{2}{*}{ Corallus hortulanus (Linnaeus, 1758) } & 1 & Ave \\
\hline & 1 & Ave e roedor \\
\hline Epicrates cenchria (Linnaeus, 1758) & 3 & Fragmentos de roedor \\
\hline \multirow[t]{2}{*}{ Eunectes murinus (Linnaeus, 1758) } & 1 & Juvenil de Bos taurus (Mammalia Artiodactyla) ${ }^{1}$ \\
\hline & 2 & Canis familiaris (Mammalia Carnivora) ${ }^{1}$ \\
\hline \multicolumn{3}{|l|}{ COLUBRIDAE } \\
\hline \multirow[t]{6}{*}{ Chironius exoletus (Linnaeus, 1758) } & 2 & Fragmentos de anuro \\
\hline & 1 & Pristimantis sp. (Anura Strabomantidae) \\
\hline & 1 & 02 Pristimantis sp. (Anura Strabomantidae) \\
\hline & 1 & Fragmentos de Hylidae \\
\hline & 2 & Hypsiboas geographicus (Anura Hylidae) \\
\hline & 2 & Scinax ruber (Anura Hylidae) \\
\hline Chironius scurrulus (Wagler, 1824) & 1 & Phyllomedusa camba (Anura Hylidae) \\
\hline \multirow[t]{2}{*}{ Dendrophidion dendrophis (Schlegel, 1837) } & 1 & Fragmentos de Pristimantis sp. \\
\hline & 1 & 02 Pristimantis sp. (Anura Strabomantidae) \\
\hline \multirow[t]{9}{*}{ Drymarchon corais (Boie, 1827) } & 2 & Rhinella marina (Anura Bufonidae) \\
\hline & 1 & Observado ingerindo um Rhinella marina (Anura Bufonidae) \\
\hline & 1 & 02 Rhinella marina (juvenis) (Anura Bufonidae) \\
\hline & 1 & Epicrates cenchria (Serpentes Boidae) + Fragmentos de anuro \\
\hline & 1 & Atractus sp. (Serpentes Colubridae) \\
\hline & 1 & Fragmentos de Ameiva ameiva (Sauria Teiidae) \\
\hline & 1 & Ameiva ameiva (Sauria Teiidae) + roedor \\
\hline & 1 & Ovo de Gallus gallus (Aves Gallifornes) \\
\hline & 1 & Roedor \\
\hline Drymoluber dichrous (Peters, 1863) & 1 & Allobates aff. marchesianus (Anura Aromobatidae) \\
\hline Masticophis mentovarius (Duméril, Bibron e Duméril, 1854) & 1 & Leptodactylus andreae (Anura: Leptodactylidae) $)^{2}$ \\
\hline Mastigodryas boddaerti (Sentzen, 1796) & 1 & Ameiva ameiva (Sauria: Teiidae) \\
\hline Oxybelis fulgidus (Daudin, 1803) & 1 & Anolis sp. (Sauria Polychrotidae) \\
\hline Pseustes poecilonotus (Günther, 1858) & 1 & Ave \\
\hline \multicolumn{3}{|l|}{ DIPSADIDAE } \\
\hline Atractus latifrons Cunha \& Nascimento, 1983 & 1 & Um carrapato (Acari, Ixodidae) \\
\hline Clelia plumbea (Wied, 1820) & 1 & Epicrates cenchria (Serpentes Boidae) \\
\hline Dipsas catesbyi (Sentzen, 1796) & 4 & Lesma (Gastropoda, Veronicellidae) \\
\hline Dipsas indica Laurenti, 1768 & 1 & 11 lesmas (Gastropoda, Veronicellidae) \\
\hline Drepanoides anomalus (Jan, 1863) & 1 & Ovo de Squamata ${ }^{2}$ \\
\hline Erythrolamprus aesculapii (Linnaeus, 1766) & 1 & Atractus latifrons (Serpentes Colubridae) \\
\hline \multirow[t]{5}{*}{ Leptodeira annulata (Linnaeus, 1758) } & 1 & Scinax ruber (Anura Hylidae) \\
\hline & 1 & Elachistocleis ovalis (Anura Microhylidae) \\
\hline & 1 & Perseguindo um Leptodactylus andreae (Anura Leptodactylidae) \\
\hline & 1 & Desova de Phyllomedusa camba (Anura Hylidae) \\
\hline & 1 & Cercosaura eigenmani (Sauria Gymnophtalmidae) ${ }^{2}$ \\
\hline Liophis almadensis. (Wagler, 1824) & 1 & Leptodactylus andreae (Anura Leptodactylidae) \\
\hline \multirow[t]{5}{*}{ Liophis reginae (Linnaeus, 1758) } & 1 & 03 Leptodactylus andreae (Anura Leptodactylidae) \\
\hline & 3 & Leptodactylus andreae (Anura Leptodactylidae) \\
\hline & 1 & Regurgitou 3 Leptodactylus andreae (Anura Leptodactylidae) \\
\hline & 1 & Fragmentos de Pristimantis sp. (Anura Strabomantidae) \\
\hline & 2 & Scinax ruber (Anura Hylidae) \\
\hline
\end{tabular}

\footnotetext{
${ }^{1}$ Observações de terceiros. ${ }^{2}$ Espécimes capturados em pitfall.
} 
Tabela 1. Continuação...

\begin{tabular}{|c|c|c|}
\hline Família/ Espécie & $\mathbf{N}$ & Conteúdo estomacal/ Observação \\
\hline \multirow[t]{3}{*}{ Liophis reginae (Linnaeus, 1758) } & 1 & Fragmentos de anuro \\
\hline & 1 & Cauda e fragmentos de girino \\
\hline & 1 & $\begin{array}{l}\text { Regurgitou um Leptodactylus lineatus (Anura Leptodactylidae) e um } \\
\text { Chiasmocleis avilapiresae (Anura Microhylidae) }{ }^{2}\end{array}$ \\
\hline \multirow[t]{6}{*}{ Oxyrhopus melanogenys (Tschudi, 1845) } & 1 & Gonatodes sp. (Sauria Sphaerodactylidae) \\
\hline & 1 & 02 Gonatodes hasemanni (Sauria Sphaerodactylidae) \\
\hline & 4 & Ameiva ameiva (Sauria Teiidae) \\
\hline & 4 & Roedor \\
\hline & 1 & Perseguindo um roedor ${ }^{1}$ \\
\hline & 1 & Fragmentos de ave (Aves Passeriformes) \\
\hline Philodryas argentea (Daudin, 1803) & 1 & Allobates aff. marchesianus (Anura Aromobatidae) \\
\hline Philodryas olfersii (Lichtenstein, 1823) & 1 & Fragmentos de roedor \\
\hline Rhinobothryum lentiginosum (Scopoli, 1785) & 1 & Mabuya nigropunctata (Sauria Scincidae) \\
\hline \multirow[t]{3}{*}{ Siphlophis worontzowi Prado, 1939} & 1 & Iphisa elegans (Sauria Gymnophthalmidae) \\
\hline & 1 & Gonatodes humeralis (Sauria Sphaerodactylidae) \\
\hline & 1 & Hemidactylus mabouia (Sauria Gekkonidae) \\
\hline Xenopholis scalaris (Wücherer, 1861) & 1 & Patas de Leptodactylus (Lithodytes) sp. (Anura Leptodactylidae) \\
\hline \multicolumn{3}{|l|}{ ELAPIDAE } \\
\hline Micrurus spixii Wagler, 1824 & 1 & M. spixii (Serpentes Elapidae) \\
\hline \multicolumn{3}{|l|}{ VIPERIDAE } \\
\hline \multirow[t]{3}{*}{ Bothrops atrox (Linnaeus, 1758) } & 1 & Fragmentos de insetos \\
\hline & 1 & Leptotyphlops sp. (Serpentes Leptotyphlopidae) \\
\hline & 1 & Roedor \\
\hline Lachesis muta (Linnaeus, 1766) & 1 & Pelos de mamífero \\
\hline
\end{tabular}

${ }^{1}$ Observações de terceiros. ${ }^{2}$ Espécimes capturados em pitfall.

\section{Discussão}

Apesar da maioria das espécies de serpentes de Espigão do Oeste predarem lagartos (55\% das espécies) e anuros (48\%) (Bernarde \& Abe 2006), nesse estudo foi encontrado maior proporção de anfíbios anuros como item alimentar das serpentes e lagartos em menor número $(15 \%$ dos registros). Na Amazônia, uma menor porcentagem de serpentes utiliza anfíbios anuros, variando de 39 a 48\% (Martins \& Oliveira 1998, Bernarde \& Abe 2006) em relação às regiões do Pantanal, sul e sudeste (59 a 81,8\% das espécies) (Strüssmann \& Sazima 1993, Yanosky et al. 1996, Marques \& Sazima 2004, Hartmann et al. 2009a, b, Zanella \& Cechin 2009). Provavelmente essas diferenças se devam à composição de espécies: onde nas regiões extra-amazônicas, ocorre maior proporção de Xenodontinae nas comunidades e grande parte das serpentes desse clado preda anuros (Cadle \& Greene 1993). Fatores históricos também foram associados com a diferença na proporção de serpentes batracófagas e saurívoras entre a Reserva Ducke (Manaus) e Santa Cecília (Equador) (Martins \& Oliveira 1998).

Os boídeos predam vertebrados, sendo os grupos registrados nesse estudo (lagartos, aves e mamíferos) conhecidos na dieta dessas serpentes (Martins \& Oliveira 1998). A predação de alguns mamíferos domésticos por Eunectes murinus, apesar de pouco documentada (e.g. Macedo-Bernarde 2006), é um evento relativamente comum nas áreas rurais (P. S. Bernarde, Obs. Pess.).

Uma dieta muito diversificada, que também inclui invertebrados, é encontrada em colubrídeos e dipsadídeos (Cadle \& Greene 1993). As espécies de Atractus são conhecidas por se alimentarem de minhocas, nesse estudo foi encontrado um carrapato no trato digestório de uma A. latifrons, evento também observado por Martins \& Oliveira (1998), podendo tratar-se de um item ocasional dessa serpente.

As serpentes pertencentes ao gênero Chironius são batracófagas e diurnas (Dixon et al. 1993). Devido à impalatabilidade de anuros pertencentes ao gênero Phyllomedusa (Sazima 1974), poucos são os relatos de predação desses animais por serpentes, sendo as espécies do gênero Chironius algumas das exceções (Dixon et al. 1993, Castanho 1996, esse estudo). Para C. exoletus foi registrada a predação sobre o anfíbio venenoso Trachycephalus mesophaeus (Rodrigues 2008). Predação sobre hilídeos é habitual nessa espécie e em espécies congenéricas que apresentam o corpo mais delgado e que forrageiam mais sobre a vegetação (Dixon et al. 1993, Marques \& Sazima 2003, 2004, esse estudo). Os anfíbios presentes na dieta das duas espécies de Chironius mencionadas acima são de hábitos noturnos, mesmo padrão observado por Pinto et al. (2008), indicando que essas serpentes caçam esses hilídeos em seus abrigos, durante o dia.

Lagartos, roedores e outras serpentes são presas habituais de serpentes pertencentes ao gênero Clelia (Martins \& Oliveira 1998, Pinto \& Lema 2002). Um espécime de C. plumbea (CRC $197 \mathrm{~cm}$, CC 37,5. $2785 \mathrm{~g}$, ) ) foi encontrado pela manhã (11 horas) predando uma Epicrates cenchria (CRC $137 \mathrm{~cm}, \mathrm{CC} 17 \mathrm{~cm}, 1885$ g, 우). Outro boídeo (Boa constrictor) havia sido também registrado para $C$. clelia (Martins \& Oliveira 1998). Apesar das serpentes do gênero Clelia serem predominantemente noturnas, atividade diurna também foi registrada para C. clelia (Duellman 1978, Martins \& Oliveira 1998).

Um espécime de Drepanoides anomalus, obtido em armadilha de interceptação e queda, apresentou um ovo de Squamata em seu conteúdo estomacal, sendo improvável que a serpente tenha ingerido o ovo no interior da armadilha e corroborando a especialização alimentar desta espécie (Martins \& Oliveira 1998).

Drymarchon corais é considerada uma serpente generalista (Vanzolini 1986, Greene 1997, França et al. 2008). Neste estudo, foi observado o hábito eurifágico dessa espécie pela presença de anuros, lagartos, serpentes, ovos de aves e roedores nos tratos digestivos dos espécimes analisados. A predação de ovos de aves é um evento pouco comum para as serpentes na região Neotropical, estando registrado para algumas espécies como o Colubridae Pseustes poecilonotus 
(Greene 1997) e o Dipsadidae Pseudoboini Rhachidelus brazili (França et al., 2008, Sawaya et al. 2008). Sapos do gênero Rhinella são venenosos, porém explorados por algumas espécies de serpentes como Xenodon rabdocephalus (Martins \& Oliveira 1998) e D. corais (Greene 1997, França et al. 2008, esse estudo). Um espécime foi observado inspecionando um buraco no chão pela manhã. Essa serpente apresenta hábito terrícola e diurno (Bernarde \& Abe 2006) e sendo a maioria das suas presas noturnas, essa espécie deve encontrá-las principalmente quando estão abrigadas.

Leptodeira annulata é considerada uma serpente batracófaga (Martins \& Oliveira 1998), sendo anfíbios anuros o principal item observado nesse estudo. Um lagarto gimnoftalmídeo (Cercosaura eignmanni) foi encontrado em um espécime capturado em armadilha de interceptação e queda. Essa serpente também é conhecida por predar ovos de Phyllomedusa (Martins \& Oliveira 1998), o que também foi registrado nesse estudo.

A serpente Liophis reginae é diurna e forrageia no chão, sendo a mais frequente nas áreas de pastagem (Bernarde \& Abe 2006), teve mais da metade de suas presas nesse estudo (56\% de 16 ítens) constituídas pelo anuro diurno e terrícola Leptodactylus andreae (Bernarde 2007), o mais abundante nesses ambientes (Bernarde \& Macedo, 2008).

Nesse estudo, anuros foram registrados como presas para outras espécies reconhecidamente batracófagas (Michaud \& Dixon 1989, Martins \& Oliveira 1998): Dendrophidion dendrophis, Drymoluber dichrous, Liophis almadensis, Philodryas argentea e Xenopholis scalaris. Além dessas, foi também registrado o consumo de um anuro Leptodactylus andreae por uma Masticophis mentovarius capturada em armadilha de interceptação e queda. A biologia dessa espécie é pouco conhecida, já tendo sido registrado roedor (Rattus rattus) em sua dieta (Dugan \& Figueroa 2008).

Duellman (1978) e Cunha \& Nascimento (1993) encontraram roedores e lagartos nos conteúdos estomacais de espécimes de Oxyrhopus melanogenys. Dos itens registrados na dieta dessa serpente, pássaro constitui o primeiro registro para esta espécie. A predação de aves é conhecida também para $O$. petola (Bernarde \& Machado 2000) e para os gêneros Boiruna e Rhachidelus dentro da Tribo Pseudoboini (Pinto \& Lema 2002, Sawaya et al. 2008). Essa serpente apresenta atividade sobre o chão e noturna (Bernarde \& Abe 2006) e preda tanto presas diurnas, possivelmente capturadas em abrigo ou repouso (lagartos Ameiva ameiva e Gonatodes hasemani; pássaro) como noturnas (roedores).

Serpentes do gênero Siphlophis são conhecidas por predarem principalmente lagartos diurnos, quando estão inativos, durante a noite (Prudente et al. 1998). Foram registradas na dieta de S. worontzowi três espécies de lagartos (Gonatodes humeralis, Hemidactylus mabouia e Mabuya nigropunctata). Um espécime de $S$. worontzowi foi encontrado durante a noite forrageando em um galho de árvore a três metros de altura em floresta e outro predando uma lagartixa (H. mabouia) a um metro e meio de altura em ambiente de pastagem também durante o período noturno. Esses dados revelam que esta espécie forrageia durante o período noturno e também sobre a vegetação e além de presas diurnas (G. humeralis e $M$. nigropunctata), preda também noturnas ( $H$. mabouia).

Os itens encontrados nas serpentes Dipsas spp. (lesmas), Erythrolamprus aesculapii (serpentes), Mastigodryas boddaerti (lagarto Ameiva), Oxibelys fulgidus (lagarto Anolis), Philodryas olfersii (roedor), Pseustes poecilonotus (aves), Rhinobothryum lentiginosum (lagarto Mabuya) e Lachesis muta (mamífero) são conhecidos para a dieta dessas espécies (Martins \& Oliveira 1998, Hartmann \& Marques 2005).

Foi registrado um evento de canibalismo em Micrurus spixii, de um macho $(\mathrm{CRC}=117,7 \mathrm{~cm} ; 300 \mathrm{~g})$ coletado ingerindo uma fêmea $(\mathrm{CRC}=70 \mathrm{~cm} ; 90 \mathrm{~g})$. Essa espécie é conhecida por predar serpentes (Martins \& Oliveira 1998), incluindo outras espécies de Micrurus. Predação de conspecíficos é um evento registrado para várias linhagens de serpentes (boídeos, dipsadídeos, elapídeos) e aparentemente é mais frequente em espécies com hábitos alimentares generalistas e ofiófagos (e.g. Engeman et al. 1996, Rivas \& Owens 2000, Hartmann \& Marques 2005).

Bothrops atrox é serpente que apresenta dieta generalista (Martins \& Oliveira 1998). Neste estudo encontrou-se uma serpente (Leptotyphlops sp.) no conteúdo de um juvenil e um roedor em um subadulto. Em um juvenil de B. atrox, foram encontrados restos de insetos na porção final do intestino, podendo tratar-se de item secundário, de algum anuro ingerido pela serpente (ver Martins \& Gordo 1993).

Em algumas espécies de serpentes, fêmeas adultas cessam ou reduzem a alimentação durante o período de gestação (Shine 1980), enquanto outras não (e.g. Aldridge \&. Bufalino 2003, Pinto \& Fernandes 2004). Nesse estudo foram encontrados anuros em três fêmeas grávidas (com ovos ou folículos vitelogênicos) de Chironius exoletus, Liophis reginae, Xenopholis scalaris, e lesma em uma Dipsas catesbyi. Essas quatro serpentes corresponderam a 23,5\% do total de fêmeas grávidas que foram analisadas. Talvez a ocorrência de conteúdos estomacais em fêmeas grávidas seja mais frequente em espécies que se alimentam de presas relativamente pequenas e de fácil digestão (e.g. anuros e lesmas).

A maioria (78\%) dos anuros encontrados nos conteúdos estomacais das serpentes correspondem a espécies que apresentam atividade no chão (e.g. Allobates, Elachistocleis, Leptodactylus e Rhinella) (Bernarde 2007, Bernarde \& Macedo 2008), enquanto que os arborícolas (e.g. Hypsiboas, Scinax e Phyllomedusa) corresponderam a apenas $22 \%$ dos conteúdos. Possivelmente esses resultados estejam relacionados ao substrato de forrageio das serpentes nessa comunidade, das quais $52 \%$ forrageiam no chão e $26 \%$ sobre a vegetação (Bernarde \& Abe 2006). A associação entre o substrato de caça de serpentes e o de atividade de suas presas também foi observado por outros autores, como Martins \& Oliveira (1998) e Albuquerque et al. (2007), que encontraram principalmente hilídeos (arborícolas) na dieta da espécie diurna e arborícola Leptophis ahaetulla, e Martins \& Oliveira (1998) e Prudente et al. (2007) que registraram anuros terrícolas como presas da serpente diurna e terrícola Dendrophidion dendrophis.

Assim como os anuros, a maioria dos lagartos encontrados nos conteúdos estomacais nas serpentes apresenta hábitos terrícolas (65\%), estando isso possivelmente também relacionado à proporção de serpentes que forrageiam no chão. Outro fator responsável pela maior freqüência de lagartos terrícolas é a elevada abundância de Ameiva ameiva nas áreas de pastagens nessa localidade, esta espécie correspondeu a $89 \%$ dos lagartos capturados nas armadilhas de interceptação e queda neste tipo de ambiente (Macedo et al. 2008)

Anfíbios anuros foi o principal item alimentar registrado, seguido de roedores e lagartos. Entretanto, a maioria das serpentes em Espigão do Oeste preda lagartos (55\% das espécies) e anuros (48\%). Na Amazônia, uma menor porcentagem de serpentes utiliza anfíbios anuros em relação às comunidades extra-amazônicas, provavelmente essas diferenças se devam à maior proporção de Xenodontinae nas comunidades extra-amazônicas e grande parte das serpentes desse clado preda anuros. A maioria dos anfíbios anuros e lagartos encontrados nos conteúdos estomacais das serpentes apresentam hábitos terrícolas, o que deve estar associado com a maioria das espécies de serpentes (52\%) forragearem sobre o chão. 


\section{Agradecimentos}

A Lílian Cristina Macedo pelo auxílio nas atividades de campo. Aos proprietários da Fazenda Jaburi Eduardo Garcia, Celso Garcia e família, pelo apoio logístico. A Carlos Alberto Bernarde e família pelo apoio logístico em Espigão do Oeste. Ao Centro de Conservação e Manejo de Répteis e Anfíbios - RAN, do IBAMA, pelas licenças de coleta concedida (Processo 02001.006649/00-60; Licenças 246/2000-DIFAS/DIREC e 021/02-RAN). À Fundação O Boticário de Proteção à Natureza pelo patrocínio concedido (Projeto $\mathrm{N}^{\circ}$ 045420002). Ao CNPq pela bolsa de produtividade em pesquisa (501927/2009-3) para PSB.

\section{Referências}

AB'SABER, A.N. 1977. Os domínios morfoclimáticos da América do Sul. Geomorfologia 52:1-21.

ALBUQUERQUE, N.R., GALATTI, U. \& DI-BERNARDO, M. 2007. Diet and feeding behaviour of the Neotropical parrot snake (Leptophis ahaetulla) in northern Brazil. J. Nat. Hist. 41(17-20):1237-1243.

ALDRIDGE, R.D. \& BUFALINO, A.P. 2003. Reproductive female common watersnakes (Nerodia sipedon sipedon) are not anorexic in the wild. J. Herpetol. 37(2):416-419.

ÁVILA-PIRES, T.C.S., HOOGMOED, M.S. \& VITT, L.J. 2007. Herpetofauna da Amazônia. In Herpetologia no Brasil II (L.B. Nascimento \& M.E. Oliveira, eds.). Sociedade Brasileira de Herpetologia, Belo Horizonte, p. 13-43.

BERNARDE, P.S. \& ABE, A.S. 2006. A snake community at Espigão do Oeste, Rondônia, Southwestern Amazon, Brazil. South Am. J. Herpetol. 1(2):102-113

BERNARDE, P.S. \& MACEDO, L.C. 2008. Impacto do desmatamento e formação de pastagens sobre a anurofauna de serapilheira em Rondônia (Brasil). Iheringia, Ser. Zool. 98(4):545-459.

BERNARDE, P.S. \& MACHADO, R.A. 2000. Oxyrhopus petola digitalis (NCN). Prey. Herpetol. Rev. 31(4):247-248.

BERNARDE, P.S. 2007. Ambientes e temporada de vocalização da anurofauna no Município de Espigão do Oeste, Rondônia, Sudoeste da Amazônia - Brasil (Amphibia: Anura). Biota Neotrop. 7(2): http://www. biotaneotropica.org.br/v7n2/pt/fullpaper?bn01507022007+pt (último acesso em 10/11/2009).

BERNARDE, P.S., FERREIRA-MARTINS, L.S. \& OLIVEIRA, J.R. 2008. Bothrocophias hyoprora (Amazonian Hog-nosed Pit Viper). Diet. Herpetol. Rev. 39(3):353.

CADLE, J.E. \& GREENE, H.W. 1993. Phylogenetic patterns, biogeography, and the ecological structure of Neotropical Snake assemblage. In Species Diversity in Ecological Communities - Historical and geographical perspectives (R.E. Ricklefs \& D. Schuluter, eds.). The University of Chicago Press, Chicago, p. 281-293.

CASTANHO, L.M. 1996. Phyllomedusa distincta (leaf-frog). Predation. Herpetol. Rev. 27(3):141.

CECHIN, S.Z. \& MARTINS, M. 2000. Eficiência de armadilhas de queda (pitfall traps) em amostragem de anfíbios e répteis no Brasil. Rev. Bras. de Zool. 17(3):729-740.

CUNHA, O.R. \& NASCIMENTO, F.P. 1993. Ofídios da Amazônia: as cobras da região Leste do Pará. Bol. Mus. Para. Emílio Goeldi., Sér. Zool. 9(1):1-191.

DIXON, J.R., WIEST, J.A. \& CEI, J.M. 1993. Revision of the tropical snake Chironius Fitzinger (Serpentes, Colubridae). Monogr. Mus. Reg. Sci. Natur. 13:1-279.

DUELLMAN, W.E. 1978. The biology of an equatorial herpetofauna in Amazonian Equador. Univ. Kansas Mus. Nat. Hist. Misc. Publ. 65:1-352.

DUGAN, E.A. \& FIGUEROA, A. 2008. Masticophis mentovarius (Neotropical whipsnake). Attempted predation and diet. Herpetol. Rev. 39(4):471.

EGLER, S.G., OLIVEIRA, M.E. \& MARTINS, M. 1996. Bothrops atrox (Common lancehead). Foraging behavior and ophiophagy. Herpetol. Rev. 27(1):22-23.
ENGEMAN, R.M., RODDA, G.H. \& RODRIGUEZ, D.V. 1996. Brown tree snake (Boiga irregularis) cannibalism. The Snake 27(2):149-152.

FRANÇA, F.G.R., MESQUITA, D.O., NOGUEIRA, C.C. \& ARAÚJO, A.F.B. 2008. Phylogeny and ecology determine morphological structure in a snake assemblage in the Central brazilian Cerrado. Copeia 1:23-38.

GREENE, H.W. 1997. Snakes: the evolution of mystery in nature. University of California Press, California.

HARTMANN, P.A. \& MARQUES, O.A.V. 2005. Diet and habitat use of two sympatric species of Philodryas (Colubridae), in south Brazil. Amp. Rep. 26(1):25-31.

HARTMANN, P.A., HARTMANN, M.T. \& MARTINS, M. 2009a. Ecologia e história natural de uma taxocenose de serpentes no Núcleo Santa Virgínia do Parque Estadual da Serra do Mar, no sudeste do Brasil. Biota Neotrop. 9(3): http://www.biotaneotropica.org.br/v9n3/pt/ fullpaper?bn03609032009+pt (último acesso em 10/11/2009).

HARTMANN, P.A., HARTMANN, M.T. \& MARTINS, M. 2009b. Ecology of a snake assemblage in the Atlantic Forest of Southeastern Brazil. Pap. Avulsos Zool. 49(27):343-360.

MACEDO, L.C., BERNARDE, P.S. \& ABE, A.S. 2008. Lagartos (Squamata: Lacertilia) em áreas de floresta e de pastagem em Espigão do Oeste, Rondônia, sudoeste da Amazônia, Brasil. Biota Neotrop. 8(1): http:// www.biotaneotropica.org.br/v8n1/pt/fullpaper?bn01108012008+pt (último acesso em 10/11/2009).

MACEDO-BERNARDE, L.C. \& BERNARDE, P.S. 2005. Bothrops atrox (Common Lancehead). Diet. Herpetol. Rev. 36(4):456.

MACEDO-BERNARDE, L.C. 2006. Eunectes murinus (Linnaeus) (Serpentes, Boidae), preying activity. PanamJAS 1(2):2.

MARQUES, O.A.V. \& SAZIMA, I. 2003. Ontogenetic color changes may strengthen suggestion about systematic affinities between two species of Chironius (Serpentes, Colubridae). Phyllomedusa 2(1):65-67.

MARQUES, O.A.V. \& SAZIMA, I. 2004. História natural dos répteis da Estação Ecológica da Juréia-Itatins. In Estação Ecológica Juréia-Itatins, ambiente físico, flora e fauna (O.A.V. Marques \& W. Duleba, eds.). Holos Editora, Ribeirão Preto, p. 257-277.

MARTINS, M. \& GORDO, M. 1993. Bothrops atrox (Common Lancehead). Diet. Herpetol. Rev. 24(4):151-152.

MARTINS, M. \& OLIVEIRA, M.E. 1998. Natural history of snakes in forests of the Manaus region, Central Amazonia, Brazil. Herpetol. Nat. Hist. 6(2):78-150.

MARTINS, M., MARQUES, O.A.V. \& SAZIMA, I. 2002. Ecological and phylogenetic correlates of feeding habits in Neotropical pitvipers (Genus Bothrops). In Biology of the vipers (G.W. Schuett, M. Höggren, M.E. Douglas \& H.W. Greene, eds.). Eagle Mountain Publishing, Utah, p. 307-328.

MICHAUD, E.J. \& DIXON, J.R. 1989. Prey itens of 20 species of the Neotropical colubrid snake genus Liophis. Herpetol. Rev. 20(2):39-41.

NASCIMENTO, P.F., BERNARDE, P.S. \& BUZZETTI, D.R.C. 2008. Bothrops atrox (Amazonian Lancehead). Diet. Herpetol. Rev. 39(3):353.

OLIVEIRA, M.E. \& MARTINS, M. 2001. When and where to find a pitviper: activity patterns and habitat use of the lancehead, Bothrops atrox, in central Amazonia, Brazil. Herpetol. Nat. Hist. 8(2):101-110.

OLIVEIRA, O.A. 2002. Geografia de Rondônia: espaço e produção. Dinâmica Ed. e Dist. Ltda, Porto Velho.

PINTO, C.C. \& LEMA, T. 2002. Comportamento alimentar e dieta de serpentes, gêneros Boiruna e Clelia (Serpentes, Colubridae). Iheringa, Sér. Zool. 92(2):9-19.

PINTO, R.R. \& FERNANDES, R. 2004. Reproductive biology and diet of Liophis poecilogyrus (Serpentes, Colubridae) from southeastern Brazil. Phyllomedusa 3(1):9-14.

PINTO, R.R., FERNANDES, R. \& MARQUES, O.A.V. 2008. Morphology and diet of two sympatric colubrid snakes, Chironius flavolineatus and Chironius quadricarinatus (Serpentes: Colubridae). Amphibia-Reptilia 29(2):149-160.

PRUDENTE, A.L.C., MASCHIO, G., SANTOS-COSTA, M.C. \& PEREIRA, C.E.Y. 2007. Reproduction and feeding aspects of Dendrophidion 
dendrophis (Schlegel, 1837) (Serpentes, Colubridae) in the Brazilian Amazon. South Am. J. Herpetol. 2(1):1-14.

PRUDENTE, A.L.C., MOURA-LEITE, J.C. \& MORATO, S.A.A. 1998. Alimentação das espécies de Siphlophis Fitzinger (Serpentes: Colubridae: Xenodontinae: Pseudoboini). Rev. Bras. Zool. 15(2):375-383.

RIVAS, J.A. \& OWENS, R.Y. 2000. Eunectes murinus (Green anaconda): Cannibalism. Herpetol. Rev. 31(1):44-45.

RODRIGUES, M.G. 2008. Chironius exoletus (Common whipsnake): prey and possible diet convergence. Herpetol. Bull. 105:41-42.

SAWAYA, R.J., MARQUES, O.A.V. \& MARTINS, M. 2008. Composição e história natural das serpentes de Cerrado de Itirapina, São Paulo, sudeste do Brasil. Biota Neotrop. 8(2):http://www.biotaneotropica.org.br/v8n2/pt/ fullpaper?bn01308022008+pt (último acesso em: 10/11/2009).

SAZIMA, I. 1974. Experimental predation on the leaf-frog Phylomedusa rohdei by the water snake Liophis miliaris. J. Herpetol. 8(4):376-377.

SHINE, R. 1980. "Costs" of reproduction in reptiles. Oecologia 46:92-100.

STRÜSSMANN, C. \& SAZIMA, I. 1993. The assemblages of the Pantanal at Poconé western Brazil: faunal composition and ecology summary. Stud. Neotr. Fauna Environ. 28(33):157-168.

TOFT, C.A. 1985. Resource partitioning in amphibians and reptiles. Copeia $1: 1-21$
TURCI, L.C.B. \& BERNARDE, P.S. 2008. Levantamento herpetofaunístico em uma localidade no município de Cacoal, Rondônia, Brasil. Bioikos 22(2):101-108.

VANZOLINI, P.E. 1986. Levantamento herpetológico da área do Estado de Rondônia sob a influência da rodovia Br-364. Ministério da Ciência e Tecnologia, Brasília, 50 p. (Relatório de pesquisa, Programa Polonoroeste/ Ecologia Animal n. 1).

VITT, L.J. 1983. Ecology of an anuran-eating guild of terrestrial tropical snakes. Herpetologica 39(1):52-66.

YANOSKY, A.A., DIXON, J.R. \& MERCOLLI, C. 1996. Ecology of the snake community at El Bagual Ecological Reserve, Northeastern Argentina. Herpetol. Nat. Hist. 4(2):97-110.

ZANELLA, N. \& CECHIN, S.Z. 2009. Influência dos fatores abióticos e da disponibilidade de presas sobre comunidade de serpentes do Planalto Médio do Rio Grande do Sul. Iheringia, Ser. Zool. 99(1):111-114.

Recebido em 16/11/09

Versão reformulada recebida em 07/03/10

Publicado em 15/03/10 